\title{
A COMPRA POR IMPULSO EM AMBIENTES ON-LINE
}

\section{RESUMO}

Este estudo tem como propósito avaliar os antecedentes e as conseqüências da compra por impulso em lojas virtuais da Internet. Para tanto, foi desenvolvido um modelo teórico que procura apresentar as relações existentes entre o ambiente de loja (site), a impulsividade individual, a circulação na loja (navegação no site) e a realização de compras impulsivas. A partir de uma pesquisa survey realizada com compradores de produtos pela Internet, por meio de modelagem de equações estruturais, foi evidenciado que o ambiente do site afeta o nível de impulsividade, a navegação no site e a ocorrência de compras por impulso, bem como se encontrou uma relação entre compra por impulso e emoções positivas. Elementos ambientais que influenciam a compra por impulso foram identificados e rotas para futuras pesquisas apresentadas, juntamente com implicações acadêmicas e gerenciais dos resultados.

\section{Filipe Campelo Xavier da Costa}

Universidade do Vale do Rio dos Sinos

\section{Juliano A. Larán}

University of Florida

\begin{abstract}
This study has as its purpose to evaluate the antecedents and consequences of impulse buying in virtual stores on the Internet. To do so, we developed a model to present the existing relationships among the web site environment, impulsivity, in-store browsing and impulse buying. From a survey design with Internet buyers, using structural equation modeling, we found that the web site environment affects the level of impulsivity, in-store browsing and the occurrence of impulse purchases, as well as a relationship between impulse purchases and positive emotions. Environmental elements influencing impulsive buying are identified and ways for future research are presented, along with academic and managerial implications of our results.
\end{abstract}

PALAVRAS-CHAVE Compra por impulso, Internet, emoções, varejo, comportamento do consumidor. KEY WORDS Impulsive buying, Internet, emotions, retailing, consumer behavior. 


\section{INTRODUÇÃo}

Estudos acerca do comportamento do consumidor têm dado grande atenção à busca de maior compreensão do processo de tomada de decisão de compra. O entendimento de suas etapas e de seus agentes influenciadores constitui-se de grande valia para os profissionais de marketing no desenvolvimento de estratégias de segmentação e posicionamento, bem como para todos elementos do composto de marketing - produto, promoção, preço e distribuição. Sabe-se hoje que um dos momentos críticos em todo processo acontece dentro do ambiente de loja, no qual a maior parte das decisões de compra ocorre (Solomon, 1999). E é dentro desse ambiente que se dá um comportamento que abrevia todo o processo decisório de compra, conhecido como compra por impulso.

O comportamento de compra por impulso ocorre quando o consumidor adquire algo de forma repentina e sem controle sobre seus atos, obtendo produtos de forma puramente emocional, desconsiderando as conseqüências do seu feito (Engel et al., 1995). Estudos indicam que esse tipo de comportamento é extremamente corriqueiro em sociedades de consumo ocidentais: segundo Welles (1986), nove de dez consumidores ocasionalmente realizam compras por impulso. Outros estudos indicam que mais de 39\% das compras em lojas de departamento e $67 \%$ das compras em supermercados ocorrem de modo impulsivo (Mowen e Minor, 1998). Entretanto, percebe-se que poucas pesquisas vêm sendo realizadas sobre o tema, sendo uma área dentro do estudo de comportamento do consumidor negligenciada pelos pesquisadores de uma forma geral (Cobb e Hoyer, 1986; Burroughs, 1996). Nos últimos anos, esforços têm sido realizados para ampliar o conhecimento acerca do tema. Beatty e Ferrel (1998) procuraram identificar os antecedentes que explicam o comportamento de compra impulsiva, relacionando variáveis situacionais e de diferenças individuais. Hausman (2000) investigou as influências de aspectos hedônicos na ocorrência de compras por impulso. Youn e Faber (2000) buscaram relacionar compra por impulso, características de personalidade e aspectos ambientais e situacionais.

Em paralelo, a emergência do varejo on-line, representada principalmente pela Internet, tem atraído a atenção nos últimos anos devido a seu potencial e suas implicações para consumidores e vendedores. O consumidor passa a usar seu tempo de forma mais eficiente, o que combinado ao maior sortimento e volume de informações, permite comparações de preços e maior conveniência pois, com as compras pela Internet, as restrições de tempo e espaço, comuns às lojas tradicionais, tendem a desaparecer (Kalakota e Whinston, 1997). Entretanto, não é sabido se esse ambiente de compra influencia o comportamento do consumidor dos mesmos modo e intensidade aos ocorridos no varejo tradicional. Eroglu et al. (2001) foram pioneiros em analisar a influência do ambiente das lojas virtuais no comportamento do consumidor; entretanto, a compra por impulso nesse canal de marketing permanece desconhecida. As investigações até então realizadas tinham como foco as compras ocorridas no varejo tradicional, fisicamente constituído. Logo, este trabalho pretende, a partir da teoria existente, estudar o comportamento de compra por impulso, bem como seus antecedentes, em um novo ambiente de compras: a Internet.

O artigo está organizado em quatro seções. A primeira parte corresponde à fundamentação teórica, ao desenvolvimento do modelo teórico e às hipóteses de pesquisa. No segundo momento, a metodologia de pesquisa é detalhada. Em seguida, o modelo proposto é avaliado e comentado. Por fim, as contribuições do estudo, suas limitações e sugestões de caminhos para novos estudos são apresentadas.

\section{FUNDAMENTAÇÃO TEÓRICA E HIPÓTESES DE PESQUISA}

A conceituação de comportamento de compra por impulso sofreu um processo evolutivo desde os primeiros estudos ocorridos na década de 1940 (Dupont apud Cobb e Hoyer, 1986). Anteriormente, considerava-se compra impulsiva toda aquisição realizada sem planejamento prévio à entrada na loja, ou seja, as compras excedentes àquelas anteriormente listadas ou reconhecidas. Em seguida, buscou-se diferenciar as compras não-planejadas - decisões de compra dentro do ambiente de loja com necessidade de aquisição já reconhecida - das compras impulsivas - necessidade de compra inexistente até a entrada na loja.

Rook (1987, p. 191) define que a compra impulsiva ocorre "quando o consumidor experimenta uma necessidade repentina, persistente e muitas vezes poderosa de comprar algo imediatamente. Esse impulso de compra é hedonicamente complexo e pode estimular conflitos de natureza emocional, com reduzida preocupação com suas conseqüências de compra". Beatty 
e Farrel (1998) completam o conceito, indicando que essa compra ocorre sem intenções prévias de adquirir algum produto ou de efetuar alguma tarefa de compra, não se constituindo em aquisição de um item lembrado no ambiente de loja. Tal conceito envolve três dimensões distintas: a circulação do consumidor na loja, a impulsividade do indivíduo e a influência dos elementos ambientais.

\section{Circulação na loja}

Entende-se como circulação dentro da loja a atividade com fins recreativos ou informativos de percorrer e examinar o ambiente sem intenção imediata de compra (Bloch, Ridgway e Sherrel, 1989). Consumidores passeiam pela loja como uma forma de entretenimento e prazer, vivenciando sentimentos positivos como contentamento (Babin, Darden e Griffin, 1994). Estudos anteriores apontam que os indivíduos que "circulam" mais tendem a realizar mais compras nãoplanejadas do que aqueles que passeiam menos (Jarboe e McDaniel, 1987). Hoch e Loewenstein (1991) corroboram essa percepção, sugerindo que o aspecto temporal - tempo disponível para circulação na loja - e a proximidade física permitem o surgimento do desejo de comprar por impulso que, por sua vez, pode se transformar em uma ação de compra. Sendo assim, percebe-se a relação existente entre a permanência dentro da loja - intensidade da circulação, ou navegação, por se tratar de estudo no varejo virtual - e a probabilidade de comprar por impulso. A partir dessa premissa, podese estabelecer a primeira hipótese de pesquisa:

$\mathrm{H}_{1:}$ Quanto maior o nível de circulação dentro da loja, maior será a incidência de compras impulsivas.

\section{Impulsividade do indivíduo}

Impulso é considerado uma necessidade forte, às vezes irresistível, com a inclinação de agir sem deliberação ou planejamento (McCown e DeSimone, 1993), sendo gerado imediatamente a partir da exposição de um certo estímulo (Wolman, 1989). Os indivíduos possuem, como traço de personalidade, níveis diferenciados de controle de seus impulsos. Hoch e Loewenstein (1991) explicam que a compra impulsiva trata do resultado da luta entre as forças psicológicas dos desejos e da força de vontade, ou seja, entre a vontade de comprar e o autocontrole, sendo que o desejo prevalece. Segundo Youn (2000), a impulsividade de compra poderia ser decomposta em duas grandes dimensões, a cognitiva e a afetiva. Na primeira, o indivíduo age sem a realização de mínimos esforços cognitivos, renden- do-se ao impulso de compra sem avaliar seus riscos e desconsiderando as possíveis conseqüências no futuro, como desapontamento, arrependimento ou problemas financeiros decorrentes da aquisição. Na dimensão afetiva, o indivíduo utiliza a compra como uma forma de reduzir seus conflitos emocionais, gerenciando seu humor; também a utiliza pois pode gerar emoções positivas, como alegria e prazer (Hausman, 2000). Supõese, assim, que os indivíduos impulsivos tendem a circular mais dentro do ambiente de loja - ou navegar em um site de venda - para o gerenciamento de seu humor e para proporcionar emoções positivas, gerando as hipóteses de pesquisa que se seguem:

$\mathrm{H}_{2}$ Os niveis de impulsividade do indivíduo influenciarão positivamente a intensidade de circulação dentro da loja /site.

$\mathrm{H}_{3}$. Quanto maior a impulsividade do indivíduo, maior será sua tendência a realizar compras por impulso.

\section{Elementos ambientais}

A relação entre o ambiente de loja e o comportamento de compra por impulso já foi sugerida na literatura (Piron, 1991). Fatores ambientais e componentes do produto - como cores, aromas, sons, texturas e localização - podem aumentar a probabilidade da ocorrência de comportamento de compra por impulso (Eroglu e Machleit, 1993; Mitchell, 1994). O uso das ferramentas do marketing mix, como promoções, displays, merchandising e propaganda, assim como as facilidades - aceitação de cartão de crédito, funcionamento 24 horas e crédito automático - tornam cada vez mais fácil para os consumidores comprarem por impulso (Rook, 1987; Rook e Fisher, 1995). Assim, a presença de elementos dessa natureza pode aumentar a permanência dos consumidores nas lojas, despertando ou gerando impulsos que levam ao ato da compra. Portanto, novas hipóteses são formuladas:

$\mathrm{H}_{4}$ : Elementos ambientais do site influenciarão positivamente na impulsividade do indivíduo.

$\mathrm{H}_{5}$ : Elementos ambientais do site influenciarão positivamente na intensidade da circulação no site.

$\mathrm{H}_{6:}$ Elementos ambientais do site influenciarão positivamente a ocorrência de compras impulsivas.

\section{Conseqüências da compra por impulso}

A ocorrência de compras por impulso pode gerar distintos tipos de conseqüências para o consumidor. Ele pode efetuar julgamentos sobre a propriedade de realizar compras impulsivas em determinada situação de consumo, gerando emoções positivas - entusiasmo, alegria, orgulho - ou negativas - culpa, irritação. 
Rook e Fisher (1995) afirmam que há a tendência de assumir o comportamento impulsivo como irracional, imaturo e perdulário, envolvendo até restrições do grupo social ao indivíduo, o que proporciona descontentamento e frustração. Em contrapartida, há consumidores que realizam a compra impulsiva predominantemente em busca do prazer de compra, tendo o item adquirido um papel secundário, de menor importância. Nesses casos, verifica-se forte correlação entre a incidência de compras impulsivas e a satisfação de necessidades de estima e auto-realização, sendo a compra uma fonte geradora de contentamento e satisfação (Hausman, 2000). As compras por impulso resultam também em emoções positivas em função do prazer gerado pela novidade ou da sensação de ter feito "um bom negócio" (Gardner e Rook, 1988). Assim, surgem as próximas hipóteses de pesquisa:

$\mathrm{H}_{7}$ :'A ocorrência de compra por impulso influencia positivamente na formação de emoções positivas.

$\mathrm{H}_{8}$ : A ocorrência de compra por impulso influencia positivamente na formação de emoções negativas.

A partir da fundamentação teórica e das hipóteses de pesquisa identificadas, a Figura 1 apresenta o modelo teórico para avaliar o impacto de antecedentes e as conseqüências da compra por impulso na Internet.

\section{MÉTODO}

Para testar empiricamente o modelo apresentado, foi efetuado um estudo descritivo de corte transver- sal, apontado por Churchill (1999) como o mais conhecido e amplamente utilizado, e que permite a obtenção de uma "fotografia" das variáveis estudadas em um momento específico no tempo, com o uso de uma amostra representativa da população-alvo (Malhotra, 2001). É importante frisar que o método possui limitações, como a impossibilidade de se fazerem inferências causais das relações propostas e a medição em apenas um momento feita uma única vez, o que não permite o acompanhamento de como se comportam os indivíduos estudados com o passar do tempo.

$\mathrm{O}$ intuito do estudo descritivo foi desenvolver e avaliar os antecedentes e as conseqüências da compra por impulso na Internet com consumidores que realizaram compras por esse meio no segundo semestre de 2001 e estão familiarizados com a navegação pela Internet. Nesta seção, são apresentadas a operacionalização do estudo, que engloba as decisões quanto ao processo de seleção de amostra e realização da coleta de dados, as medidas empregadas, as técnicas estatísticas utilizadas e uma breve caracterização da amostra em estudo.

\section{Procedimentos de amostragem e de coleta de dados}

A amostra deste estudo é de 2.634 consumidores on-line, residentes em 22 estados brasileiros, que responderam a um questionário on-line entre 21 e 28 de fevereiro de 2002. O banco de dados e o desenvolvimento do questionário em sua versão on-line foram feitos pela empresa E-bit, especializada na realização de avaliações de lojas virtuais e tem como clientes os

Figura 1 - Modelo de antecedentes e conseqüências da compra por impulso na Internet.

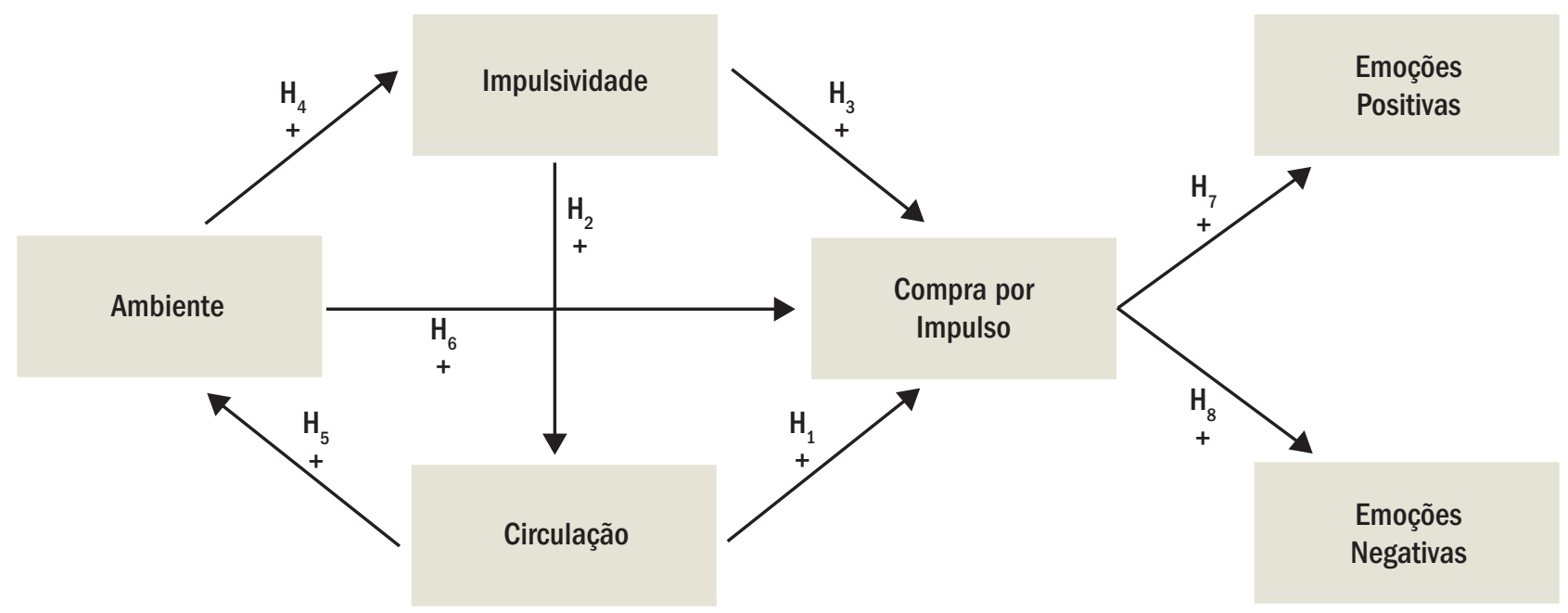


principais sites de venda do país - Submarino, Americanas, Ponto Frio, Saraiva etc. A partir de um banco de dados com mais de 20 mil nomes, foi sorteada uma amostra aleatória de 10 mil usuários. Para esses usuários, foram enviadas mensagens eletrônicas convidando para participar do estudo em um endereço eletrônico específico, sendo que a amostra de envio foi dividida em duas sub-amostras do mesmo questionário, mas com ordem diferenciada das questões para evitar o viés da seqüência das questões. A taxa de resposta foi de $26,3 \%$, e os respondentes receberam como estímulo pontos da E-bit para concorrerem a sorteios promovidos pela empresa.

\section{Medidas}

As medidas utilizadas neste trabalho foram retiradas de estudos realizados por Beatty e Ferrel (1998) para as dimensões "circulação na loja" - dez itens - e compra por impulso - dois itens. Empregou-se a escala de impulsividade de Youn (2000), que é composta de 24 itens e subdividida em três construtos de natureza afetiva - sentimento de urgência de compra, geração de emoções positivas com a compra e gerenciamento do humor - e dois construtos cognitivos desconsideração do futuro e ação sem deliberação. No caso dessas medidas, foi utilizada uma escala de concordância bietápica, na qual o entrevistado indicava se concordava ou não com a afirmação e, em seguida, apontava o grau de concordância /discordância em três posições. As emoções foram avaliadas a partir das medidas desenvolvidas por Watson et al. (1988), com 12 afetos - oito positivos e quatro negativos - em uma escala de intensidade de sete posições. Para os componentes ambientais, foram identificados 19 itens de loja virtual a partir da realização de 30 entrevistas em profundidade com consumidores on-line na cidade de Porto Alegre e dos trabalhos de Youn e Faber (2000) e Eroglu et al. (2001). Os elementos foram avaliados a partir de uma escala tipo Likert de cinco posições, variando entre 1 - nenhuma importância - a 5 - total importância.

A partir das escalas acima mencionadas, foi elaborado o questionário de pesquisa. Para a apreciação de seu conteúdo, foi conduzida a avaliação por parte dos juízes, com cinco professores experts na área de comportamento do consumidor de diversas universidades brasileiras. O instrumento foi analisado sob vários aspectos, identificando-se problemas de escalas, itens, ordenamento de questões e instruções. Modificações no instrumento foram realizadas e foi feito um segundo pré-teste com 20 usuários da Internet a partir de entrevistas pessoais, com a versão impressa do questionário para avaliar a compreensão do instrumento. Alguns ajustes foram realizados e foi então confeccionada a versão on-line do instrumento com o auxílio da equipe do E-bit, principalmente na construção da página na qual o questionário on-line foi disponibilizado. Por fim, partiu-se para o terceiro pré-teste, com a aplicação do questionário pela Internet em 120 casos, gerando a versão final do instrumento.

\section{Processos estatísticos de análise}

Foram empregados métodos estatísticos - como estatísticas descritivas - e técnicas de análise multivariada - como análise fatorial e modelagem de equações estruturais. Para tanto, os softwares SPSS 10.0 e AMOS $4^{\oplus}$, foram utilizados.

Como o propósito deste trabalho é o teste de hipóteses e a avaliação da significância estatística do modelo de compra por impulso, foi empregada a modelagem de equações estruturais. Segundo Hair Jr. et al. (1998), essa abordagem possibilita o uso de uma estrutura mais complexa de variáveis observadas manifestas - e não-observáveis - latentes -, bem como a análise de seus inter-relacionamentos.

O processo de análise foi iniciado pelo modelo de mensuração, quando foram avaliadas a confiabilidade $\mathrm{e}$ as validades convergente e discriminante dos construtos utilizados. Nesse momento, a análise fatorial confirmatória foi empregada, pois ela permite a especificação dos relacionamentos entre as variáveis manifestas e os construtos latentes, avaliando a validade e a confiabilidade das medidas empregadas para operacionalizar o modelo (Hair Jr. et al., 1998). Primeiro, os construtos foram analisados individualmente, verificando-se sua unidimensionalidade - validade convergente. Em seguida, agrupamentos foram feitos para verificar a validade discriminante dos construtos. Realizadas essas análises, o modelo estrutural foi estimado empiricamente, assim como as relações entre os construtos, sendo avaliados os índices de ajustamento e a significância dos parâmetros estimados. Esse procedimento é conhecido como two-step approach, sugerido por vários pesquisadores na literatura da área (Anderson e Gerbing, 1988; Schumacker e Lomax, 1996; Kline, 1998).

\section{Caracterização da amostra}

A amostra estudada apresenta um predomínio dos compradores on-line da Região Sudeste, correspondendo a $70,6 \%$ dos entrevistados - somente o Estado 
de São Paulo corresponde a 44,6\%. A média de idade foi de 32 anos - d.p. $=9,76$ - e 52,5\% são mulheres. Dos respondentes, $30 \%$ possuem renda familiar mensal inferior a R \$ 2 mil e 36,6\% entre R 2 mil e R 4 mil mensais. Dos compradores, $82,3 \%$ são heavy buyers, pois realizam compras pela Internet pelo menos três vezes ao mês, principalmente para aquisição de CDs - 75,8\% - , livros e revistas - 68,5\% - e equipamentos eletrônicos - 40,1\%. Uma parcela expressiva dos entrevistados admite sentir necessidade de realizar compras por impulso - 30,9\% - e $26,9 \%$ dos respondentes indicaram realizar compras por impulso com regularidade.

\section{ANÁLISE DOS DADOS E RESULTADOS}

Primeiramente, são apresentados os resultados da análise fatorial exploratória utilizada para purificar as medidas. Em seguida, são mostrados os resultados da análise fatorial confirmatória feita para validar o modelo de medidas. Por fim, são reportados os resultados da análise do modelo estrutural usado para testar as relações entre os construtos bem como os índices de ajustamento e considerações sobre modelos teóricos rivais.

\section{Modelo de mensuração}

O primeiro passo foi identificar as variáveis que explicam a maior parte da variância de cada fator. Sendo esta a primeira aplicação do modelo no Brasil, foi utilizada análise fatorial exploratória para que se retirassem as variáveis que não explicam uma porção de variância significativa em cada um dos construtos. Esse procedimento só não foi aplicado em relação ao "ambiente", que foi dividido em quatro fatores que representavam dimensões de interesse em relação a esse construto. Os quatro fatores foram definidos igualmente a partir de análise fatorial exploratória, quando foram identificados os seguintes fatores ambientais: confiança, apresentação do site, preço e tempo da transação.

A amostra foi dividida em duas sub-amostras $\mathrm{n} 1=1.335 ; \mathrm{n} 2=1.299-$, sendo a primeira empregada como amostra de calibração para o modelo final. Como critérios para identificar os melhores indicadores de cada um dos construtos foram selecionadas as variáveis com comunalidades iguais ou superiores a $0,5 \mathrm{e}$ valores de carga fatorial iguais ou superiores a 0,5. Esse processo resultou no seguinte número de itens excluídos em cada construto: circulação - sete itens -, impulsividade - 16 itens - e emoções positivas - dois itens. Com a redução do número de itens, os alfas de Cronbach (Tabela 1) das escalas purificadas podem ser considerados aceitáveis, com exceção do construto "circulação", cujo alfa ficou abaixo de 0,6, considerando o limite de aceitação.

Após a purificação das medidas por meio de análise fatorial exploratória, a validade do modelo de mensuração total foi avaliada com o uso de análise fatorial confirmatória na amostra de validação $-\mathrm{n}=1.299$. As medidas de confiabilidade do construto utilizadas seguiram sugestão de Fornell e Larcker (1981) e Hair Jr. et al. (1998), excedendo o mínimo aceitável de 0,5 (Tabela 1). As cargas fatoriais dos indicadores em seus construtos são significantes ao nível $\mathrm{p}<0,05$, e as correlações entre variáveis de um mesmo construto foram altas, indicando validade convergente.

Para avaliação da validade discriminante, foi empregado um teste robusto sugerido por Fornell e Larcker (1981), em que a variância média extraída para cada construto deve ser maior que o quadrado do coeficiente de correlação entre o construto dado e todos os demais. Os resultados foram satisfatórios, pois o maior quadrado do coeficiente de correlação

Tabela 1 - Médias dos construtos, desvios-padrão e medidas de confiabilidade.

\begin{tabular}{|l|l|l|c|c|c|}
\hline \multicolumn{1}{|c|}{ CONSTRUTO } & MÉDIA & DESVIO-PADRÃO & $\begin{array}{c}\text { COEFICIENTE } \\
\text { ALFA }\end{array}$ & $\begin{array}{c}\text { VARIÂNCIA } \\
\text { EXTRAÍDA }\end{array}$ & $\begin{array}{c}\text { CONFIABILIDADE } \\
\text { DO CONSTRUTO }\end{array}$ \\
\hline Ambiente & 3,85 & 1,00 & 0,88 & 0,79 & 0,92 \\
\hline Impulsividade & 2,56 & 1,59 & 0,90 & 0,45 & 0,86 \\
\hline Circulação & 4,00 & 1,49 & 0,53 & 0,68 & 0,84 \\
\hline Compra por Impulso & 2,51 & 1,49 & 0,78 & 0,89 & 0,94 \\
\hline Emoções Positivas & 4,26 & 1,63 & 0,88 & 0,57 & 0,88 \\
\hline Emoções Negativas & 2,49 & 1,54 & 0,88 & 0,84 & 0,93 \\
\hline
\end{tabular}


(Tabela 2) entre dois construtos foi de 0,24 , ao passo que a variância média extraída variou de 0,45 a 0,89.

Os índices de ajustamento do modelo completo foram aceitáveis $-\chi^{2}=2.030,79 ; \mathrm{GL}=317 ; \mathrm{p}<0 ; \mathrm{GFI}=0,89$; AGFI $=0,87 ; \quad$ IFI $=0,9 ; \quad$ TLI $=0,89 ; \quad$ CFI $=0,9$; RMSEA $=0,065$. Apesar do qui-quadrado ter sido significante, o tamanho da amostra sugere que seja analisada a razão qui-quadrado /graus de liberdade. Essa razão foi de 6,4, acima do máximo sugerido por Hair Jr. et. al. (1998). No entanto, assumindo o expressivo tamanho da amostra, foram considerados os índices de ajustamento mais robustos, como o IFI, o CFI e o TLI. Esses índices comprovam o bom ajustamento do modelo de medidas, que reflete cada construto conceitual e teoricamente, com confiabilidade, validade convergente e validade discriminante.

\section{Modelo estrutural}

Após balizar a adequação do modelo de medidas, o modelo estrutural foi analisado por meio de seus índices de ajustamento, da magnitude e da significância dos parâmetros estimados. A Tabela 3 apresenta os parâmetros padronizados das relações entre os construtos e seus respectivos t-values. O modelo estrutural foi estimado com o uso do software AMOS 4, ${ }^{\circledR}$. O ajuste do modelo proposto inicialmente é satisfatório, apresentando índices como $\chi^{2}=3.873,15 ; \mathrm{GL}=317 ; \mathrm{p}<0$; IFI=0,9; TLI=0,89; CFI=0,9; RMSEA=0,065. Como o objetivo da pesquisa era testar esse modelo em um novo contexto, alguns modelos rivais foram utilizados para dar suporte à discussão da aplicabilidade e validade do modelo inicialmente proposto pelos autores.

O primeiro modelo rival - Rival 1 - tem como premissa que o ambiente de compra não influencia diretamente a ocorrência de compras impulsivas, mas contribui para que haja uma maior circulação dentro do ambiente de loja, bem como pode acionar comportamentos impulsivos do indivíduo, levando assim a uma compra por impulso. Beatty e Ferrel (1998) admitem que elementos da atmosfera de loja podem levar o indivíduo a "passear" mais no site, estando mais exposto aos estímulos e, dessa forma, suscetível de realizar compras impulsivas. A compra por impulso per se não seria diretamente influenciada por elementos ambientais, pois sua ocorrência dependeria de uma predisposição a se comportar de forma impulsiva - nível de impulsividade do indivíduo - e de sua permanência no site - circulação. Percebe-se não ter havido uma melhora nos índices de ajustamento como um todo (Tabela 3), e um teste de diferença qui-quadrado realizado apontou uma modificação significante nesse índice. Conforme apontado por Kline (1998, p. 133), uma diferença significativa nesse teste, quando se está eliminando um caminho estrutural do modelo, significa que o modelo foi simplificado em demasia, devendo-se adotar, assim, o modelo original. Logo, o modelo alternativo Rival 1 não traz aperfeiçoamento ao modelo originalmente proposto.

O segundo modelo alternativo - Rival 2 - assume que a impulsividade do indivíduo não exerce influência direta sobre o nível de compras efetuadas por impulso, mas seria mediada pela intensidade da circulação realizada pelo indivíduo na loja e, por sua vez, na ocorrência de compras impulsivas. Rook e Hoch (1985), assim como Bellenger e Korgaonkar (1980), sugerem que compradores impulsivos tendem a "programar" suas compras a partir de sua circulação nas lojas, sem identificar a priori o que deve ser buscado ou propriamente adquirido. Logo, a impulsividade individual não ocasionaria compras impulsivas sem um elevado grau de circulação dentro do site e de permanência dentro do ambiente de compra. O modelo alternativo aqui proposto apresentou um decréscimo nos índices de ajustamento e, como no modelo Rival 1, uma diferença significativa no teste qui-quadrado, o que indicou uma simplificação demasiada, não tra-

Tabela 2 - Matriz de correlação.

\begin{tabular}{|l|l|l|l|l|l|l|}
\hline CONSTRUTO & AMB & IMP & CIRC & CI & EP & EN \\
\hline AMB & 1,00 & & & & \\
\hline IMP & 0,09 & 1,00 & & & \\
\hline CIRC & 0,12 & 0,03 & 1,00 & & \\
\hline CI & 0,16 & 0,49 & $-0,02$ & 1,00 & \\
\hline EP & 0,23 & 0,33 & 0,05 & 0,33 & 1,00 & \\
\hline EN & 0,07 & 0,11 & 0,04 & 0,04 & 0,07 & 1,00 \\
\hline
\end{tabular}


zendo aperfeiçoamento para o modelo original deste estudo.

Devido à ausência de melhora no ajustamento dos modelos rivais testados, o modelo apresentado na Figura 1 foi adotado como o modelo final de estimativa das relações entre os construtos. A Tabela 4 apresenta os parâmetros padronizados, seus valores $t$ e significância.

Seis dos oito relacionamentos propostos apresentaram significância estatística no sentido hipotético. Não foram confirmadas as hipóteses da relação entre a compra por impulso e as emoções negativas, que não apresentaram uma relação significante, e da relação entre circulação e compra por impulso, que estão relacionadas no sentido inverso à hipótese dos autores. Uma possível explicação para o fato é que a circulação navegação no ambiente virtual - é comprometida pela redução de todo ambiente de loja a uma tela de computador (Eroglu et al., 2001). O computador retira uma série de apelos de natureza tangível e influentes na experiência de compra, desde o próprio ambiente físico da loja, até a presença de outros clientes e vendedores (Baker, 1986; Bitner, 1992), que, no varejo tra- dicional, influencia compras por impulso. A atividade de busca e aquisição torna-se mais objetiva, com estímulos restritos às informações relevantes à decisão de compra a ser empreendida (Eroglu et al., 2001), o que pode explicar a ausência de impacto da navegação circulação - na compra impulsiva.

Dentre os achados do estudo, comprovou-se a influência do construto "ambiente" sobre a impulsividade individual de compra, possibilitando afirmar que os estímulos presentes na loja virtual podem despertar a impulsividade individual, e esta gera compras impulsivas. No que se refere às conseqüências da compra impulsiva, evidenciou-se seu efeito na geração de emoções positivas. A natureza hedônica da atividade de compra por impulso (Hausman, 2000), que pode ser empregada para o gerenciamento do humor do indivíduo (Youn, 2000) ou até mesmo para reduzir conflitos emocionais (Bellenger e Korgaonkar, 1980), gera sentimentos positivos, como alegria, entusiasmo e orgulho. Os resultados confirmam o estudo de Gardner e Rook (1988), indicando que as emoções decorrentes de compra por impulso são predominantemente positivas. Por outro lado, a compra por impulso pode le-

Tabela 3 - Índices de ajustamento do modelo original e dos modelos rivais.

\begin{tabular}{|l|c|c|c|}
\hline \multicolumn{1}{|c|}{ ÍNDICES DE AUSTAMENTO } & MODELO ORIGINAL & MODELO RIVAL 1 & MODELO RIVAL 2 \\
\hline$\chi 2$ (Qui-quadrado) & $2.030,79(p=0,00)$ & $2.045,95(p=0,00)$ & $2.447,02(p=0,00)$ \\
\hline GL (Graus de Liberdade) & 317 & 318 & 318 \\
\hline CFI (Índice de Ajustamento Comparativo) & 0,90 & 0,90 & 0,88 \\
\hline TLI (Índice Tucker-Lewis) & 0,89 & 0,89 & 0,86 \\
\hline IFI (Índice de Ajustamento Incremental) & 0,90 & 0,90 & 0,90 \\
\hline RMSEA (Erro de Aproximação Quadrado Médio Raiz) & 0,065 & 0,065 & 0,072 \\
\hline
\end{tabular}

Tabela 4 - Estimativas dos parâmetros padronizados.

\begin{tabular}{|l|c|r|r|c|}
\hline \multicolumn{1}{|c|}{ CAMINHO ESTRUTURAL } & COEFICIENTE PADRONIZADO & $\boldsymbol{P}$ & VALOR T & HIPÓTESES \\
\hline Circulação $\rightarrow$ Compra por Impulso & $-0,05$ & $=0,05$ & $-1,96$ & H1 \\
\hline Impulsividade $\rightarrow$ Circulação & 0,07 & $<0,01$ & 2,85 & H2 \\
\hline Impulsividade $\rightarrow$ Compra por Impulso & 0,60 & $<0,01$ & 25,68 & H3 \\
\hline Ambiente $\rightarrow$ Impulsividade & 0,04 & $=0,05$ & 1,96 & H4 \\
\hline Ambiente $\rightarrow$ Circulação & 0,14 & $<0,01$ & 5,21 & H5 \\
\hline Ambiente $\rightarrow$ Compra por Impulso & 0,12 & $<0,01$ & 5,60 & H6 \\
\hline Compra por Impulso $\rightarrow$ Emoções Positivas & 0,43 & $<0,01$ & 17,04 & H7 \\
\hline Compra por Impulso $\rightarrow$ Emoções Negativas & 0,03 & $=0,13$ & 1,52 & H8 \\
\hline
\end{tabular}


var a um processo de dissonância no pós-compra, quando o consumidor tende a racionalizar os aspectos negativos dessa compra, evitando assim emoções negativas.

A seguir são discutidas as principais contribuições teóricas e gerenciais dos resultados obtidos na pesquisa, bem como suas limitações e sugestões de futuras pesquisas.

\section{DISCUSSÃO}

Sob a ótica acadêmica, o modelo proposto traz contribuições na compreensão do comportamento de compra por impulso em uma série de aspectos: 1) os estudos até então realizados estavam focados no entendimento dos antecedentes do comportamento por impulso (Rook e Hoch, 1985; Rook, 1987; Beatty e Ferrel, 1998; Youn, 2000; Hausman, 2000), não abordando as conseqüências da compra impulsiva de uma forma integrada; 2) aos estudos de compra por impulso foi agregado o componente ambiental, principalmente em razão da avaliação de seu impacto nos antecedentes e na própria ocorrência de compra impulsiva, até então desconhecidos; 3 ) a compra por impulso foi reconhecida como geradora de emoções positivas, em consonância com o estudo de Gardner e Rook (1988); 4) foi feita a relação entre a impulsividade do indivíduo e o nível de circulação no ambiente de loja; e 5) foi construído de um modelo integrativo do comportamento de compra impulsiva no campo do comércio eletrônico, canal ainda carente de estudos sobre o comportamento do consumidor.

A partir da avaliação do modelo desenvolvido, é possível constatar que, dentre os antecedentes de maior impacto sobre a ocorrência da compra impulsiva, a impulsividade individual do consumidor destaca-se. De modo semelhante ao encontrado por Beatty e Ferrel (1998), esse traço de personalidade constitui-se, a partir dos dados coletados neste estudo, como o principal antecedente sobre as compras impulsivas, seja em lojas fisicamente constituídas ou em sites de venda na Internet. A impulsividade do consumidor não é expressa apenas sob a forma de atos de compra com reduzida capacidade avaliativa, mas também como uma incapacidade momentânea de postergar recompensas com intuito de satisfação imediata.

Baumeister (2002) sugere que o comprador impulsivo enfrenta dificuldades em manter seu autocontrole por três fatores: dificuldade de estabelecer objetivos claros de consumo, incapacidade de exercer controle sobre seu orçamento pessoal e desgaste emocional decorrente dos esforços para controlar sua impulsividade. Segundo o autor, a impulsividade do consumidor decorre muito mais das falhas de autocontrole do que das influências que o meio pode exercer. Neste estudo, de modo consoante ao proposto por Baumeister, percebe-se que essa característica individual demonstrou influência mais expressiva do que os elementos do ambiente de loja ou a intensidade de circulação na mesma. Cabe aqui lembrar até que ponto a personalidade do indivíduo propicia a ocorrência de comportamentos impulsivos, sejam eles de compra ou não. Segundo Freud (apud Rook e Hoch, 1985), a personalidade tem origem no conflito entre o princípio do prazer e o principio da realidade, no qual o indivíduo encontra, de forma inconsciente, um equilíbrio entre a dicotomia do racional e do emocional. Indivíduos com maiores tendências ao predomínio do id (emoção), são mais permissivos e propensos à ocorrência de comportamentos de natureza impulsiva. Logo, pode-se constatar neste estudo que a impulsividade, decorrente de um traço de personalidade, influencia decisivamente a realização de compras por impulso.

Outro aspecto que deve ser considerado na apreciação do modelo deste artigo diz respeito à natureza dos bens adquiridos prioritariamente em lojas virtuais, o que Baumeister (2002) denominou de "produtos hedônicos", ou seja, bens adquiridos para proporcionar prazer ao indivíduo. Assumindo que o processo de compra em lojas virtuais é destituído de uma série de estímulos e influências ambientais que as lojas fisicamente constituídas possuem e, por essa razão, tem uma natureza mais "fria" ou "técnica", a maioria das aquisições realizadas pela Internet, até o momento, diz respeito aos produtos destinados a gerar prazer, como livros e CDs. Assim, é possível entender que esses tipos de compras geram uma expectativa de emoções positivas, como alegria e entusiasmo.

O estudo desenvolvido também oferece contribuições para os profissionais de marketing das empresas ligadas ao comércio eletrônico. Os resultados sugerem que o ambiente do site exerce impacto significativo na impulsividade do comprador e na frequência de compras por impulso. Dentre os componentes de maior expressão na influência da realização de compras impulsivas, resultantes da análise exploratória, estão as dimensões "facilidade de realização da transação de compra", "concessão de benefícios de preço" - des- 
contos, promoções - e, por fim, "credibilidade e confiança em relação à loja virtual".

Tratando-se da Internet, a dificuldade de reproduzir a experiência de compra tradicional em um ambiente eletrônico e remoto restringe a ocorrência de comportamentos de compra idênticos aos ocorridos em lojas físicas (Eroglu et al., 2001; Klein, 2001). A intangibilidade desse tipo de compra, associada à baixa interatividade existente, comparada a existente em uma loja física, naturalmente torna a experiência virtual mais simplificada e, talvez, menos estimulante.

Segundo Klein (2001), o grande desafio para a expansão do comércio eletrônico ao consumidor é o aumento da interatividade da experiência de compra, o que foi denominado pela autora como "telepresença". Somente a partir do momento em que o consumidor se sentisse no ambiente virtual como um ator no processo, não apenas um espectador de uma apresentação de informações padronizadas, é que a interatividade do ambiente físico poderia ser substituída pela oferecida em contextos virtuais, aumentando a adoção desse tipo de canal de comercialização.

Além disso, estudos comprovam que a legibilidade do site da loja virtual, ou seja, a apresentação e sua facilidade para ser "navegado", bem como sua capacidade de estimulação no comprador, são fundamentais para a intenção de compra (Wang et al., 2002). Caso o usuário não sinta estímulos do ambiente para desenvolver sua atividade de busca em uma loja virtual informações, rapidez, layout -, provavelmente a experiência de compra será insatisfatória. Sendo assim, a probabilidade de o ambiente despertar a impulsividade do indivíduo seria reduzida, e também a ocorrência de compras impulsivas.

Desse modo, pode-se apontar que a construção de um site de vendas que possua grande interatividade para o usuário, além de legibilidade e capacidade de estimulação, contribui para uma experiência de compra mais próxima da atividade desempenhada em ambientes físicos, tornando a compra menos "técnica". Havendo uma maior interatividade e personalização na atividade de busca e aquisição, como propiciado por empresas como a Amazon, que apresenta os produtos comprados por consumidores que adquiriram o item que ora se procura, é possível gerar impulsos de consumo e, conseqüentemente, compras impulsivas. Além disso, o emprego de apelos racionais na comunicação da loja virtual pode despertar o interesse e a impulsividade do consumidor, desde a maior conveniência de compra - loja aberta 24 horas por dia"-, até a disponibilidade de estoques com maior variedade de produtos ou preços mais reduzidos que os oferecidos pelas lojas físicas.

Por fim, é importante reconhecer que um dos fatores que afastam potenciais consumidores da Internet é o elevado risco percebido de realizar transações à distância, muitas vezes com provedores que não estão presentes no mundo físico (Klein, 2001; Eroglu et al., 2001; Wang et al., 2002). Empresas que possuem atuação nos dois ambientes com a mesma marca - Livraria Cultura, Ponto Frio, Lojas Americanas, por exemplo - levam vantagem em relação àquelas que estão apenas na Internet. Para essas últimas, é fundamental construir marcas fortes, com bons serviços e comunicação off-line intensa, para gerar reconhecimento de marca. O comportamento de compra por impulso somente ocorrerá nas lojas virtuais com a mesma intensidade que nas lojas físicas a partir do momento em que não existir mais desconfiança ou temores quanto à realização de compras pela Internet.

As relações encontradas no modelo, em sua maioria, são estatisticamente significantes porém fracas, tanto no que se refere aos parâmetros do modelo quanto às correlações entre os construtos, o que pode ter sido causado pelo tamanho expressivo da amostra, que tende a detectar qualquer relação mínima entre os construtos do modelo. Os resultados deixam margem à discussão sobre a significância prática dessas relações, que poderiam ter sido encontradas apenas devido ao tamanho da amostra. Segundo Kline (1998), um parâmetro padronizado inferior a 0,1 pode ser considerado fraco, mas, de acordo com o autor, esses limiares devem ser ajustados de acordo com a área de pesquisa em que são aplicados. Além disso, tendo sido o modelo baseado em estudos teóricos anteriores, cujos resultados suportam relações semelhantes, acredita-se que os relacionamentos apontados sejam válidos, o que não torna dispensáveis investigações futuras com o intuito de se verificar a existência de relacionamentos mais fortes.

\section{Limitações do estudo e sugestões para futuras pesquisas}

As contribuições proporcionadas por este estudo precisam ser analisadas a partir das limitações inerentes ao método de pesquisa. Inicialmente, a inexistência de um número razoável de estudos empíricos sobre o comportamento de compra por impulso, bem como 
de medidas amplamente testadas e consolidadas, faz com que os resultados do estudo sejam avaliados como preliminares. É necessária a realização de um maior número de investigações empíricas sobre compra impulsiva a fim de que as escalas e os modelos construídos possam ser aperfeiçoados e consolidados.

Apesar de a amostra empregada ser expressiva, foi gerada a partir de um banco de dados de uma empresa, o que restringe a generalização dos resultados. Adicionalmente, a concessão de estímulos ao respondente pode ser criticada por gerar um viés no estudo.

O estudo apresentado, por se constituir em um estudo de corte transversal, operacionalizado a partir de uma survey, não permite inferir relações de causa e efeito, que podem ser realizadas apenas com pesquisas de natureza experimental (Schumacker e Lomax, 1996). Logo, o uso de experimentos, com a manipulação de variáveis e a inferência de relações causais, é fortemente recomendável em novas pesquisas sobre o comportamento de compra por impulso.

Algumas questões emergem do estudo, exigindo novas investigações, como a relação entre os antecedentes e as conseqüências da compra impulsiva no varejo tradicional. Outro tópico de interesse é a comparação do comportamento de compra impulsiva em lojas físicas e lojas virtuais, buscando identificar a importância do ambiente de loja na impulsividade e na circulação. Por fim, a ampliação do estudo das conseqüências da compra impulsiva, além da avaliação das emoções, é recomendável em futuras pesquisas.

\section{Artigo recebido em 28.01.2003. Aprovado em 26.06.2003.}

\section{Referências bibliográficas}

ANDERSON, J. C. e GERBING, D. W. Structural equation modeling in practice: a review and recommended two-step approach. Psychological Bulletin, n. 103, p. 411-23, 1988.

BABIN, B., DARDEN, W. e GRIFFIN, M. Work and/or fun: measuring hedonic and utilitarian shopping value. Journal of Consumer Research, v. 20, p. 644-56, Mar. 1994

BAKER, J. The role of environment in marketing services: the consumer perspective. In: The services marketing challenge: integrated for competitive advantage, 1986. Proceedings. American Marketing Association, 1986. p. 79-84.

BAUMEISTER, R. F. Yielding to temptation: self-control failures, impulsive purchasing, and consumer behavior. Journal of Consumer Research, v. 28, p. 670-6, Mar. 2002
BEATTY, S. e FERREL, E. Impulse buying: modeling its precursors. Journal of Retailing, v. 74, n. 2, 1998.

BELLENGER, D. e KORGAONKAR, P. Profiling the recreational shopper Journal of Retailing, v. 58, p. 58-81, Spring 1980.

BITNER, M. J. Servicescapes: the impact of physical surroundings on customers and employees. Journal of Marketing, v. 56, p. 57-71, Apr. 1992.

BLOCH, P., RIDGWAY, N. e SHERREL, D. Extending the concept of shopping: an investigation of browsing activity. Journal of Academy of Marketing Science, v. 17, p. 13-21, Winter 1989.

BURROUGHS, J. E. Product symbolism, self meaning, and holistic matching: the role of information processing in impulsive buying. In: Advances in Consumer Research, 1996. Proceedings. Association for Consumer Research, 1996. p. 463-9.

COBB, C. J. e HOYER, W. D. Planned versus impulse purchase behavior. Journal of Retailing, v. 62, n. 4, Winter 1986.

CHURCHILL JR., G. Marketing research: methodological foundations. Orlando : Dryden Press, 1999

EROGLU, S. e MACHLEIT, K. Atmospherics factors in the retail environment: sights, sounds and smells. In: Advances in Consumer Research, 1993. Proceedings. Association for Consumer Research, 1993.

EROGLU, S., MACHLEIT, K. e DAVIS, L. Atmospherics qualities of online retailing - a conceptual model and implications. Journal of Business Research, v. 54, p. 177-84, 2001

FORNELL, C. e LARCKER, D. F. Evaluating structural equation models with unobserved variables and measurement error. Journal of Marketing Research, v. 18, p. 39-50, Feb. 1981.

GARDNER, M. e ROOK, D. W. Effects of impulse purchases on consumers' affective states. In: Advances in Consumer Research, 1988. Proceedings. Association for Consumer Research, 1988. p. 127-30.

HAIR JR., J. F., ANDERSON, R. E., TATHAM, R. L. e BLACK, W. C. Multivariate data analysis. $5^{\text {th }}$. ed. New Jersey : Prentice Hall, 1998.

HAUSMAN, A. A multi-method investigation of consumer motivations in impulse buying behavior. Journal of Consumer Marketing, v. 17, p. 403-19, 2000.

HOCH, S. e LOEWENSTEIN, G. Time-inconsistent preferences and consumer self-control. Journal of Consumer Research, v. 17, p. 492-507, 1991.

IYER, E. Unplanned purchasing: knowledge of shopping environment and time pressure. Journal of Retailing, v. 65, p. 40-57, Spring 1989.

IYER, E. e AHALAWAT, S. Deviations from a shopping plan: when and why do consumers not buy items as planned. In: Advances in Consumer Research, 1987. Proceedings. Association for Consumer Research, 1987. p. 246-50.

JARBOE, G. R. e McDANIEL, C. D. A profile of browsers in regional shopping malls. Journal of the Academy of Marketing Science, v. 15, p. 4653, Spring 1987. 
JEON, J. O. An empirical investigation of the relationship between affective states, in-store browsing, and impulse buying. 1990. Doctoral Dissertation University of Alabama, Tuscaloosa.

KALAKOTA, R. e WHINSTON, A. Electronic commerce: a manager's guide. Addison-Wesley, 1997. KLEIN, L. R. Creating virtual experiences in computer-mediated environments. In: EXPERIMENTIAL E-COMMERCE CONFERENCE, 2001, East Lansing. Proceedings. East Lansing: Michigan State University, 2001. CD-ROM.

KLINE, R. B. Principles and practice of structural equation modeling. New York : Guilford Press, 1998.

MALHOTRA, N. K. Pesquisa de marketing: uma orientação aplicada. $3^{\mathrm{a}}$ ed. São Paulo : Bookman, 2001.

MCCOWN, W. e DESIMONE, P. Impulses, impulsivity, and impulsive behaviors: a historical review of a contemporary issue. In: The impulsive client - theory, research and treatment. Washington: American Psychological Association, 1993

MITCHELL, D. For the smell of it all: functions and effects of olfaction in consumer behavior. In: Advances in Consumer Research, 1994. Proceedings. Association for Consumer Research, 1994.

MOWEN, J. C. e MINOR, M. Consumer behavior. $5^{\text {th }}$. ed. Upper Saddle River : Prentice Hall, 1998.

O'GUINN, T. e FABER, R. Compulsive buying: a phenomenological exploration. Journal of Consumer Research, v. 16, p. 147-57, Sept. 1989.

PIRON, F. Defining impulse purchasing. Advances in Consumer Research, v.18, p. 509-14. 1991.

ROOK, D. W. The buying impulse. Journal of Consumer Research, v. 14, p. 189-99, Sept. 1987.

ROOK, D. W. e FISHER, R. Normative influences on impulsive buying behavior. Journal of Consumer Research, v. 22, p. 305-13, Dec. 1995.
ROOK, D. W. e HOCH, S. J. Consuming impulses. In: Advances in Consumer Research, 1985. Proceedings. Association for Consumer Research, 1985. p. 23-7.

SCHUMACKER, R. E. e LOMAX, R. G. A beginner's guide to structural equation modeling. New Jersey : Lawrence Erbaum Associates, 1996.

SOLOMON, M. R. Consumer behavior - buying, having and being. 4 $^{\text {th }}$. ed. Upper Saddle River : Prentice Hall, 1999.

WANG, L., BAKER, J. e WAGNER, J. The role of atmospherics in e-tailing. In: AMA SUMMER EDUCATOR'S CONFERENCE, 2002. Proceedings. American Marketing Association, 2002.

WATSON, D., CLARK, A. e TELLEGEN, A. Development and validation of brief measures of positive and negative affect: the Panas scales. Journal of Personality and Social Psychology, v. 54, p. 1063-70, June 1988.

WELLES, G. We are in the habit of impulsive buying. USA Today, May $21^{\text {th }}, 1986$.

WEUN, S., JONES, M. e BEATTY, S. A parsimonious scale to measure impulse buying tendency. In: AMA EDUCATORS'S CONFERENCE Enhancing Knowledge Development in Marketing, 1997. Proceedings. American Marketing Association, 1997.

WOLMAN, B. B. Dictionary of behavioral science. New York: Academic Press, 1989.

YOUN, S. The dimensional structure of consumer buying impulsivity: measurement and validation. 2000. Doctoral Dissertation - University of Minnessota.

YOUN, S. e FABER, R. Impulse buying: its relation to personality traits and cues. In: Advances in Consumer Research, 2000. Proceedings. Association for Consumer Research, 2000. p. 179-85.

\title{
Filipe Campelo Xavier da Costa
}

Professor da Universidade do Vale do Rio dos Sinos. Doutor em Administração pela FEA-USP. Interesses de pesquisa na área de marketing, especialmente em comportamento do consumidor.

E-mail: fcampelo@mercado.unisinos.br

Endereço: Centro de Ciências Econômicas. Universidade do Vale do Rio dos Sinos. Av. Unisinos, 950. São Leopoldo, RS. CEP 93.022-000.

\author{
Juliano A. Larán \\ Doutorando em Marketing na University of Florida. Mestre em Administração /Marketing pela EA-UFRGS. \\ Interesses de pesquisa na área de marketing, especialmente em comportamento do consumidor. \\ E-mail: julianolaran@hotmail.com \\ Endereço: Department of Marketing. University of Florida. Gainesville, FL. 32611-7155. P.0. Box 117155. USA.
}

